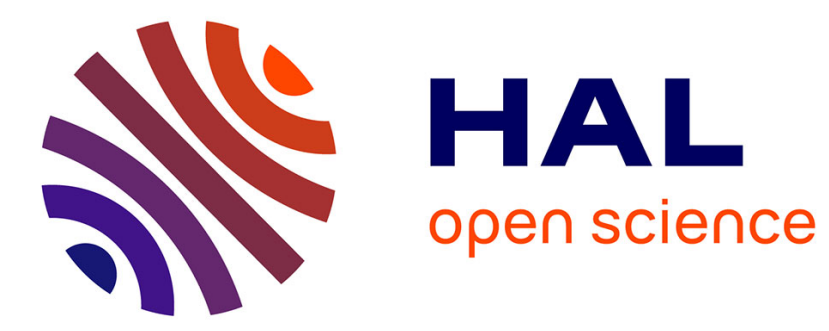

\title{
Qualitative Evaluation of a Method for Information Systems Engineering Processes
}

\author{
Charlotte Hug, Nadine Mandran, Agnes Front, Dominique Rieu
}

\section{To cite this version:}

Charlotte Hug, Nadine Mandran, Agnes Front, Dominique Rieu. Qualitative Evaluation of a Method for Information Systems Engineering Processes. Research Challenges in Information Science, May 2010, France. pp.257-268, 10.1109/RCIS.2010.5507385 . hal-00542831

\section{HAL Id: hal-00542831 \\ https://hal.science/hal-00542831}

Submitted on 21 Feb 2012

HAL is a multi-disciplinary open access archive for the deposit and dissemination of scientific research documents, whether they are published or not. The documents may come from teaching and research institutions in France or abroad, or from public or private research centers.
L'archive ouverte pluridisciplinaire HAL, est destinée au dépôt et à la diffusion de documents scientifiques de niveau recherche, publiés ou non, émanant des établissements d'enseignement et de recherche français ou étrangers, des laboratoires publics ou privés. 


\title{
Qualitative Evaluation of a Method for Information Systems Engineering Processes
}

\author{
Charlotte Hug, Nadine Mandran, Agnès Front, Dominique Rieu \\ Laboratory of Informatics of Grenoble \\ Grenoble University \\ Grenoble, France \\ \{Charlotte.Hug, Nadine.Mandran, Agnes.Front, Dominique.Rieu\}@imag.fr
}

\begin{abstract}
This paper presents a qualitative evaluation of a method for building information systems engineering processes. It includes the description of the evaluated method, the profiles of the selected subjects and the set up protocol. We also describe the results that allowed us to improve the method.
\end{abstract}

Information systems engineering processes method; qualitative evaluation; experiment; protocol

\section{INTRODUCTION}

Any method that is meant to be usable and beneficial has to be evaluated. Among others, it is important to evaluate a method's usage and usability, that is to say how users will use it and the ease and efficiency with which they will use it. In order to properly evaluate a method, a rigorous protocol has to be set. The protocol has to explicate the different objectives of the evaluation and the hypotheses to confirm, describing the more accurately as possible the manipulations to carry out and the questions to ask to the subjects. The quality of the results and their exploitation directly depends on the quality of the protocol.

Evaluation is an essential step in a method's life. However, this topic is not often dealt as it is a complex task, requiring time and some resources. Moreover, this sharp skill is not much hold by information systems researchers but rather by quality engineers. Every protocol has to be specifically designed for the evaluated method, and only a few approaches have been proposed in order to help the evaluation conception. In [6], Moody proposes a framework to evaluate IS design methods based on other works [7, 5]. Actually, this framework can be used to evaluate any kind of methods, as we used it to evaluate a method to build IS engineering processes.

In this paper, we then focus on the qualitative evaluation of a complex method to build information systems engineering processes, called ProMISE [3]: we focus as much on the protocol we developed to carry out the experiments as on the results. In this qualitative evaluation, we decided to evaluate the method with two different kinds of subjects: a focus group of modelling experts and interviews of information systems domain industrialists. The objectives of the experiments were to measure the usage and usability of the proposed method; however the experiments are different according to the focus group and the industrialists.
Section II describes the bases of the ProMISE method to build information systems engineering processes. Section III briefly presents related works to method evaluation and section IV describes the focus group experiment. Section V presents the industrialists experiment. These two last sections are organised in the same way; we first present the objectives of the evaluation and our hypothesis, we then describe the protocol of the experiment and present the results. Finally, section VI concludes this paper.

\section{PRESENTATION OF THE METHOD}

The proposed method - ProMISE (Process Metamodelling for Information Systems Engineering) [3] - aims helping method engineers defining process models for information systems engineering (ISE) according to the specificities and constraints of their organization. The method is divided in three steps:

- Creation of the process metamodel: the method guides method engineers building the process metamodel including all the knowledge of information systems engineering processes of their organization. This step is based on a conceptual graph and concepts definitions.

- Creation of the process models: once the metamodel is defined, the process models can be instantiated from it, using a formalism base in order to adequately represent the processes.

- Following of the projects: the process models are instantiated for each information systems engineering project. The managers can then regularly follow each step of their projects in consistency with the defined process models using monitoring documents.

Figure 1 presents the three steps of the method and the different artefacts used and produced. We describe in detail the three steps and the artefacts in the next sub-sections. 


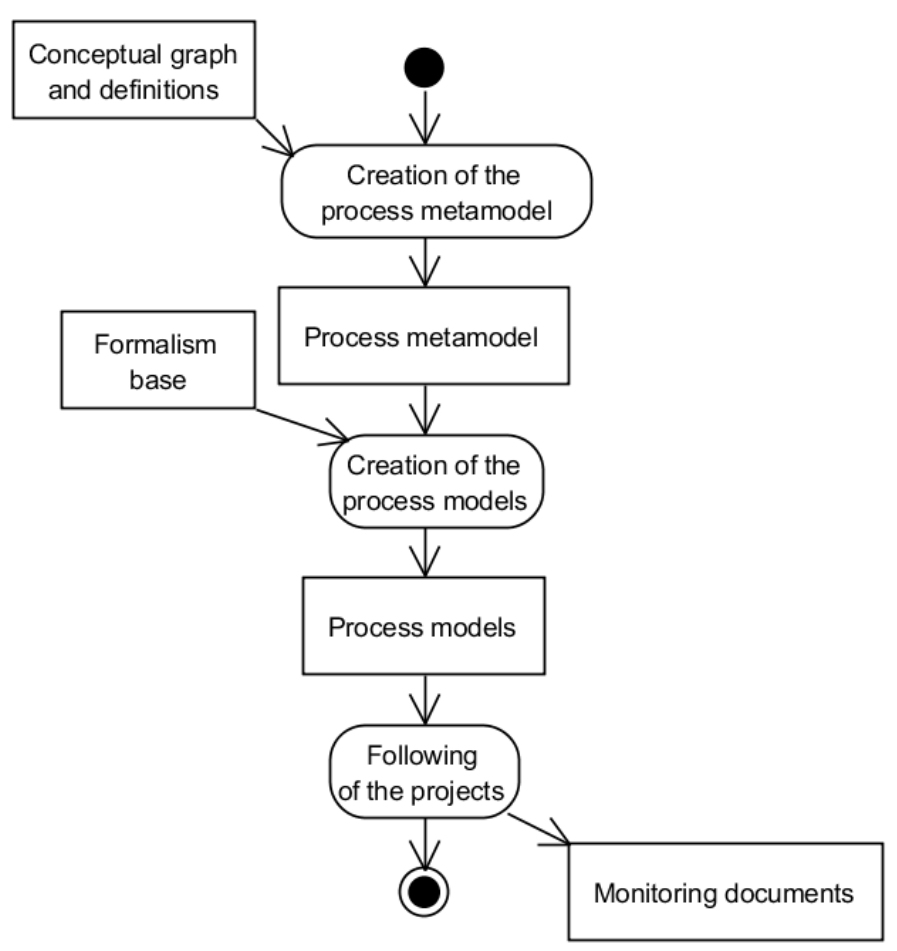

Figure 1. The process of the ProMISE method.

\section{A. Creation of the process metamodel}

The creation of the process metamodels is supported by a conceptual graph in which method engineers will select concepts to build their process metamodels. The conceptual graph is composed of concepts from existing process metamodels [4] and of relations of three types: precision (to refine a concept) concretization (to define a concept at more concrete level) and completeness (to complete a concept). The conceptual graph allows guiding method engineers in selecting the concepts according to what they want to do with their process metamodel under construction: refining (precision), completing (completeness) or concretizing (concretization) the concepts of their process metamodel under construction. The selection of a concept automatically results in the addition of classes and associations in the process metamodel under construction. Thus, the process metamodels are built as method engineers select concepts in the conceptual graph. The construction of the process metamodel it-self is hidden from the method engineers as it is a complex task described in detail in $[3,4]$. Figure 2 presents an extract of the conceptual graph with the concepts method engineers can select as Intention that represents the objectives of the ISE process. Intention can be refined by the Intention Composition concept that represent the sub-objectives of the ISE process, or concretized by the Work Product concept that is something that is produced, used or modified by a work unit during the ISE process. Work Product can be refined by Work Product Composition (sub-set of work products) and Work Product Category (set of work products that share the same properties) concepts.

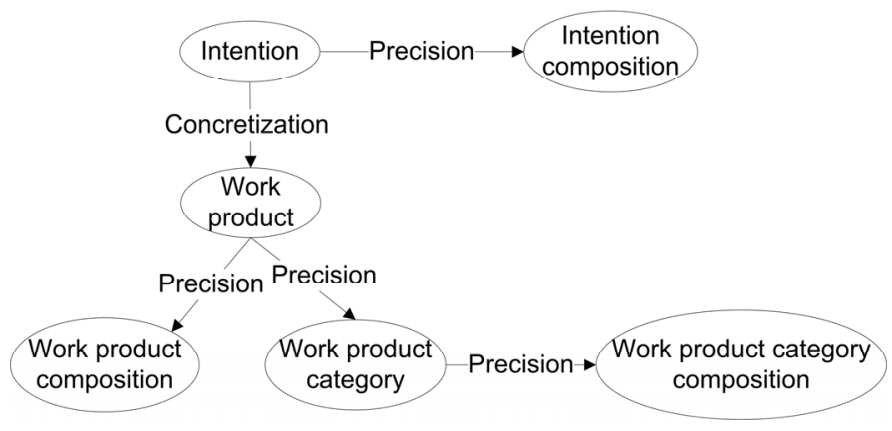

Figure 2. Extract of the conceptual graph.

In order to help method engineers selecting the concepts, the method proposes definitions, synonyms and examples of each concept of the conceptual graph. Relations are defined between the concepts in order to facilitate their selection (as a path in the conceptual graph) and to check the coherence of the selected concepts (restricted to a certain path).

The process metamodels that can be built using the method include many points of view of the information systems engineering processes: activity, product, decision, context and strategy. $[3,4]$ present these points of view in detail that come from existing process metamodels. We classified these points of view in two abstraction levels: the intentional level encompasses the context and strategy points of view and represents the objectives of the information systems engineering processes; the operational level includes the activity, product and decision points of view and represents the actions required to concretize these objectives. The knowledge of these notions is not compulsory to understand the paper.

Figure 3 presents an extract of a process metamodel that can be built using the method. It corresponds to the concepts of the conceptual graph presented in Figure 2. Intention is at the intentional level, Work Product and Work Product Category are at the operational level. The dependency "concretizes" between Work Product and Intention corresponds to the concretization link in the conceptual graph between the same concepts. The aggregations on the classes come from the concepts "X composition" defined in the conceptual graph that lead to a composition or aggregation on a class of the process metamodels under construction.

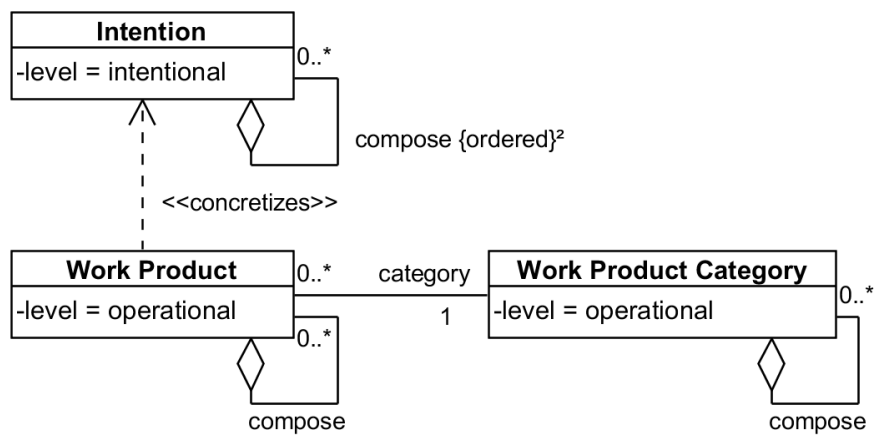

Figure 3. Extract of a process metamodel under construction. 


\section{B. Creation of the process models}

The first step in the creation of the process models is the selection of the formalism. For each class and association of the created process metamodel, method engineers have to select a graphic element, thanks to the formalisms base we propose [3]. The formalism is selected according to the habits of the organization; it's important to let method engineers selecting the most adequate icons to their environments or to the user's experience. Figure 4 presents an example of a selected formalism for each class and association of the previous process metamodel.

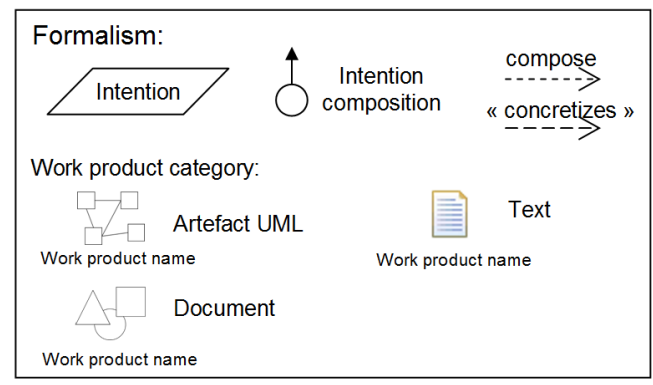

Figure 4. Example of a selected formalism.

Then, method engineers can instantiate their process metamodel using the selected formalism in order to model the information systems engineering processes of their organisation. Each process model is instantiated according to the organisation's ISE processes and must be compliant with its metamodel. Figure 5 shows an extract of one of the process models that can be instantiated from the previous process metamodel using the selected formalism. For example, "Define the level of impact..." is an intention, and is composed of two sub-intentions that are concretized by a work product. "Simplified requirements" is a work product belonging to the Text work product category and "Actors diagram" is a work product belonging to the Artefact UML work product category.

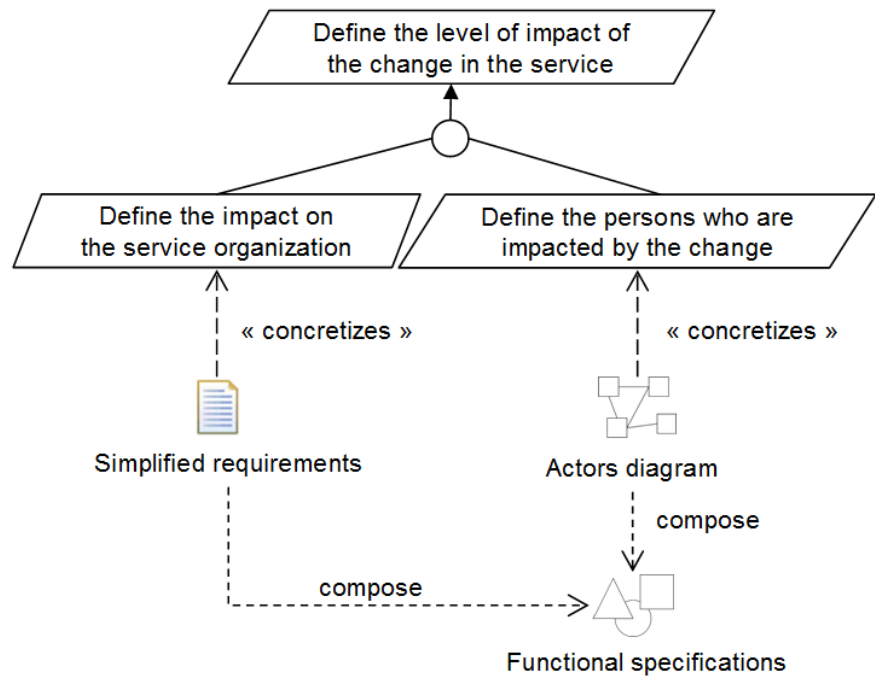

Figure 5. Example of a process model.

The instantiation of a metamodel is not an easy task. However, method engineers do not start from scratch as there are already ISE processes used in their organisations. Some or all of these ISE processes might no be modelled. Nevertheless, method engineers dispose of the material that has to be represented. Our method guides method engineers through the different step of the creation of a process metamodel but does not help them finding what they have to model. One of our next objectives is to complete the ProMISE method using contingency criterions in order to advise method engineers in the selection of concepts and their instantiation.

\section{Following of the projects}

During a particular information systems engineering project, project managers will use the defined process models in order to guide and follow the advance of the work. Each project leads to a particular instantiation of the defined process models.

\section{Prototype}

A prototype has been developed to support the method. It allows method engineers selecting concepts via their definition or the conceptual graph (see the interface in Figure 6). The tool automatically builds the process metamodels that can be exported in XMI. The next step will consist of developing the instantiation of the process metamodels built within the same tool, in order to cover the whole method.

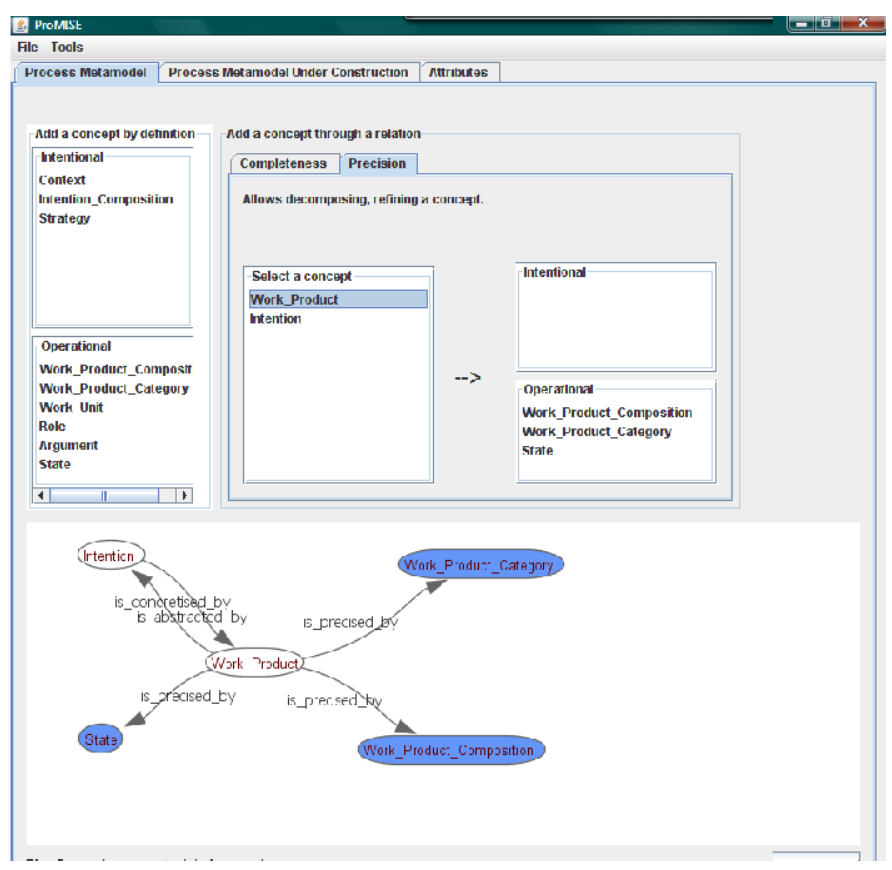

Figure 6. Interface of the ProMISE tool.

Our objectives are then to evaluate: the method, the understanding of the conceptual graph that is part of the method and the prototype. Next section presents works related to the evaluation domain.

\section{RELATED WORKS}

Evaluating a method is a hard task. In [5], the author tackled the problem of measuring the quality of a system. 
Davis proposed the Technology Acceptance Model (TAM) that is based on two characteristics:

- $\quad$ Perceived usefulness, defined as "the degree to which a person believes that using a particular system would enhance his or her job performance" [5],

- Perceived ease of use, defined as "the degree to which a person believes that using a particular system would be free of effort." [5]

TAM is also pertinent for evaluating methods. Moody [6] integrated TAM with the Methodological Pragmatism [7] which states that an objective validation of a method should be measured by its efficiency and effectiveness with which it achieves its objectives. The Method Evaluation Model includes then (see Figure 7):

- The actual efficiency that is the effort required to apply a method,

- The actual effectiveness that represents the degree to which a method achieves its objectives,

- The perceived ease of use,

- The perceived usefulness,

- The intention of use that is the extent to which a person intends to use a particular method,

- The actual usage: the extent to which a method is used in practice.

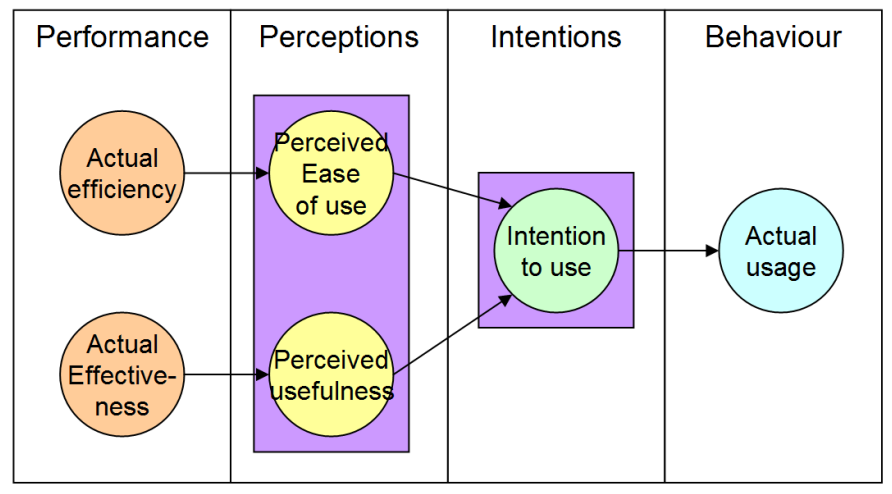

Figure 7. The Method Evaluation Model.

Others definitions are widely used in experimentations as usage [9] and usability [8]. Usage is "the act or a manner of using" [1]. Usability is "the effectiveness, efficiency and satisfaction with which specified users achieve specified goals in particular environments" [2].

Usability includes perceived effectiveness and perceived ease of use. In this paper, we focus on the method perception of the subjects as it is a qualitative evaluation. In order to measure the actual efficiency and effectiveness, quantitative experiments have to be conducted as these constructs are based on tangible data called input measures, such as execution duration, cost (efficiency) and output measures such as the produced models quality (effectiveness). Intention to use was also measured. Actual usage cannot be evaluated in an experimental context as the method needs to be practiced during some time before getting tangible data. paper.

We will use the terms usage and usability in the rest of the

\section{FOCUS GROUPE EXPERIMENT}

The first experiment has been conducted on a focus group composed of ten experts in modelling. A focus group is " $a$ group of people brought together to give their opinions on a particular issue or product" [1]. Here are the focus group characteristics:

- $\quad$ Average age: 34 years old $(\min 26, \max 47)$,

- Sex: 6 women, 4 men,

- Post: 6 PhD students, 3 teaching and research staff, 1 engineer trainee.

The subjects of the focus group did not know the ProMISE method before the experiment but they were quite used to use information systems engineering method such as Symphony, BPM, RUP or AFNOR X50-X51.

\section{A. Objectives}

The goals of the focus group experiments were to measure:

- The understanding of the conceptual graph, and

- The usage and usability of the method.

As the development of the prototype wasn't over when we begun the experiment, we could neither use nor evaluate it with the focus group. Although, the protocol strictly corresponds to the prototype conditions, that is to say, the construction of the process metamodel it -self is not carried out by the users.

\section{B. Hypothesis}

Different hypotheses allow answering the objectives. If a hypothesis is validated, then the objective is achieved. Table I presents the different objectives and the corresponding hypotheses of the experiment.

\section{TABLE I. OBJECTIVES AND HYPOTHESES}

\begin{tabular}{|c|c|}
\hline $\begin{array}{l}\text { Objectives to } \\
\text { measure }\end{array}$ & Hypotheses \\
\hline $\begin{array}{l}\text { Understanding of the } \\
\text { conceptual graph }\end{array}$ & $\begin{array}{l}\text { The subjects will build the same conceptual } \\
\text { graph as ours (1) }\end{array}$ \\
\hline \multirow{5}{*}{ Usage of the method } & $\begin{array}{l}\text { The conceptual graph is useful to build } \\
\text { information systems engineering process } \\
\text { metamodels (2) }\end{array}$ \\
\hline & $\begin{array}{l}\text { The conceptual graph covers the main concepts } \\
\text { of information systems engineering process (3) }\end{array}$ \\
\hline & $\begin{array}{l}\text { The process metamodel is useful to define } \\
\text { process models }(5)\end{array}$ \\
\hline & $\begin{array}{l}\text { Many iterations are needed to get a complete } \\
\text { process metamodel (6) }\end{array}$ \\
\hline & $\begin{array}{l}\text { The virtuous circle process metamodel/process } \\
\text { model allows innovation }(7)\end{array}$ \\
\hline Usability of method & The method is easy to use and is efficient (4) \\
\hline
\end{tabular}




\section{Protocol}

The experiment was divided in two parts. The subjects worked in pair.

In the first experiment, the subjects had to build a conceptual graph by using the concepts and relations definitions. They had to set the concepts using post-its on a white board and draw the relations between the post-its using erasable pens of three different colours, one colour by relation type. This first experiment lasted 3 hours and its goal was to measure the understanding of the conceptual graph.

In the second experiment, the subjects had to use our method according to a case study. The case study described in natural language an extract of an information system engineering process involving concepts of the intentional and operational abstraction levels. The subjects had to select the corresponding concepts in our conceptual graph highlighting them in a given paper copy. In parallel, the experimenter built the corresponding process metamodel under construction: this was done by hand during the experimentation but it could have been done using the prototype. Once the subjects had selected all the needed concepts in the conceptual graph (that is to say built their first version of the process metamodel), they could instantiate the metamodel using the formalisms base, to get a process model representing the process described in the case study. During the instantiation, it was possible to come back to the selection of other concepts in the conceptual graph, if concepts were missing. Then, the process metamodel under construction was updated and the instantiation could continue. The experiment stopped when the subjects got their complete process model. This second experiment lasted 2 hours and has been conducted within the week after the first experiment. Its objectives were to measure the usage and usability of the method.

We asked the subjects to fill two forms. The first form was filled at the beginning of the first experiment. The subjects had to indicate their habit of information systems development and their use of design and development methods. This first form was mainly used to identify the characteristics of the focus group and to put the subjects in the mood.

The second form was filled at the end of the second experiment. This second form was important to analyze and measure the usage and the usability of the method through the subject's answers.

We now describe the results of these experiments according to the objectives to measure.

\section{Results}

Phrases italicized correspond to the verbatim of the subjects (s1 to $\mathrm{s} 10)$.

\section{1) Understanding of the conceptual graph}

Figure 8 shows a conceptual graph obtained by a pair of subjects at the end of the first experiment. Each pair of subjects succeeded into grouping the concepts by point of view around the main concepts such as Work unit, Product and Context. However, the relations were not completely understood; each pair of subjects interpreted and used them differently: "Is
Abstraction the opposite of Completeness?" (s.1), "We have floundered a lot with the relations" (s.9).

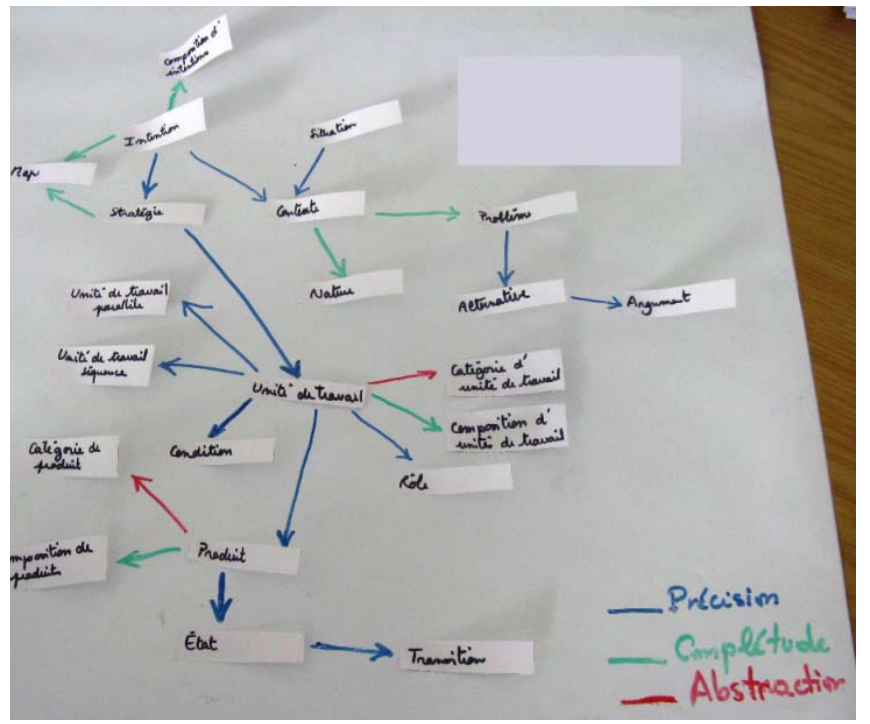

Figure 8. Conceptual graph built by a pair of subjects.

This experiment showed that the comprehension of the concepts was not easy for all the subjects because of the ambiguity of their definitions. The difficulty laid in the fact that the used vocabulary was very specific to the domain, here information systems engineering: "I do not come from information systems. In project management, the strategy concept is something defined in the long term in an organization. If the experiment is conducted with people from mechanic, there would be bias due to the business domain" (s.5).

Furthermore, the concepts definitions used words that were not defined as concepts which seemed to be a problem for the subjects: "We had some difficulties to identify 'action' cited in the definitions, but we realized it was Work unit" (s.2).

The concepts definitions list begun with the intentional concepts, the subjects declared that it would have been easier if the operational concepts came first in the list.

Hypothesis (1) is so partly validated. Those results brought us to improve the concepts and relations definitions. The new definitions have next been presented to the subjects for another validation. This second evaluation allowed us to consolidate the definitions. The new definitions are now clearly understandable and validated.

\section{2) Usage of the method}

The items in Figure 9 allowed us to evaluate the usage of the method during the second experiment.

Is the conceptual graph complete?

Was the metamodel essential to create the expected process model? Why?

Did the iteration metamodel/model allow you to find new ideas? Would you have preferred to directly modify the process metamodel? Why?

Figure 9. Items about the usage of the method. 
Figure 10 shows the concepts selected by a pair of subjects in our version of the conceptual graph.

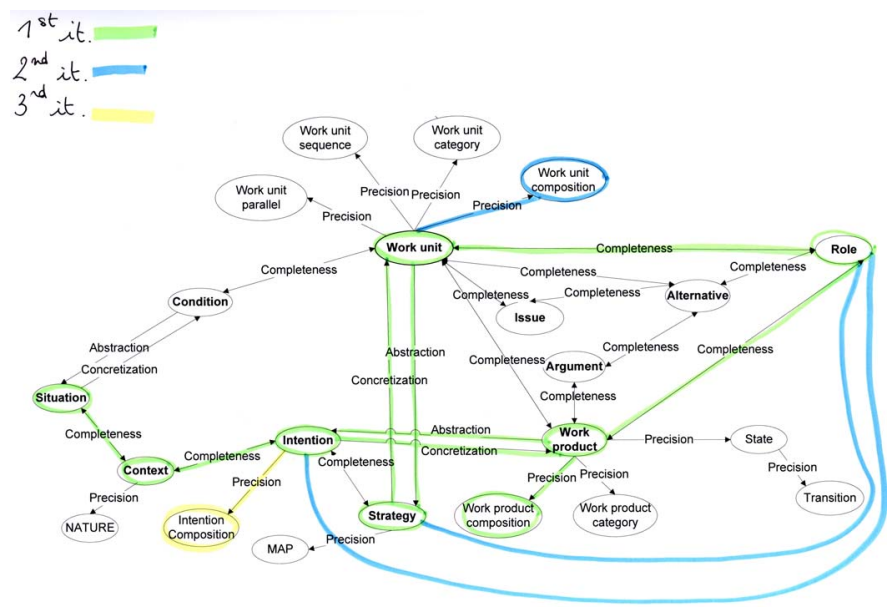

Figure 10. Selection of the concepts in the conceptual graph by a pair of subjects.

This exercise showed that the selection of the concepts in the conceptual graph was easy for 3 subjects and difficult for 4 subjects, out of 10 . This mitigated result is due to the concepts definitions that were ambiguous, as recounted in the previous section. However, all the subjects thought the method could avoid them doing mistakes because "it is rigorous" (s. 10), it "decreases the complexity of modelling" (s. 7) and "it defines a framework assuring the completeness and the coherence of the models" (s. 2). Hypothesis (2) is then partly validated.

During this exercise, the subjects did not add new concepts: the covering of the conceptual graph is then complete. Nevertheless, a pair of subjects had added a relation between Role and Intention and Role and Strategy. We then have to improve our conceptual graph to take into account these changes. Hypothesis (3) is partly validated.

9 subjects out of 10 thought that the process metamodel was useful to instantiate the process model (the remaining subject is not used to work with metamodelling): the metamodel is "an important guide, without it, it would be more difficult to design the process model" (s.3) and it "allows fixing the possibilities and the boundaries of the process models" (s.6). All the subjects were satisfied of the exercise and the process models they obtained. The process metamodel allows to easily and rigorously instantiate process models: " $B y$ using $a$ predefined and clear set of concepts, we can check if our model is coherent" (s.1) and "the UML class diagram is easier to instantiate" (s.6). Hypothesis (5) is then validated. Figure 11 presents the process models instantiated by a pair of subjects.

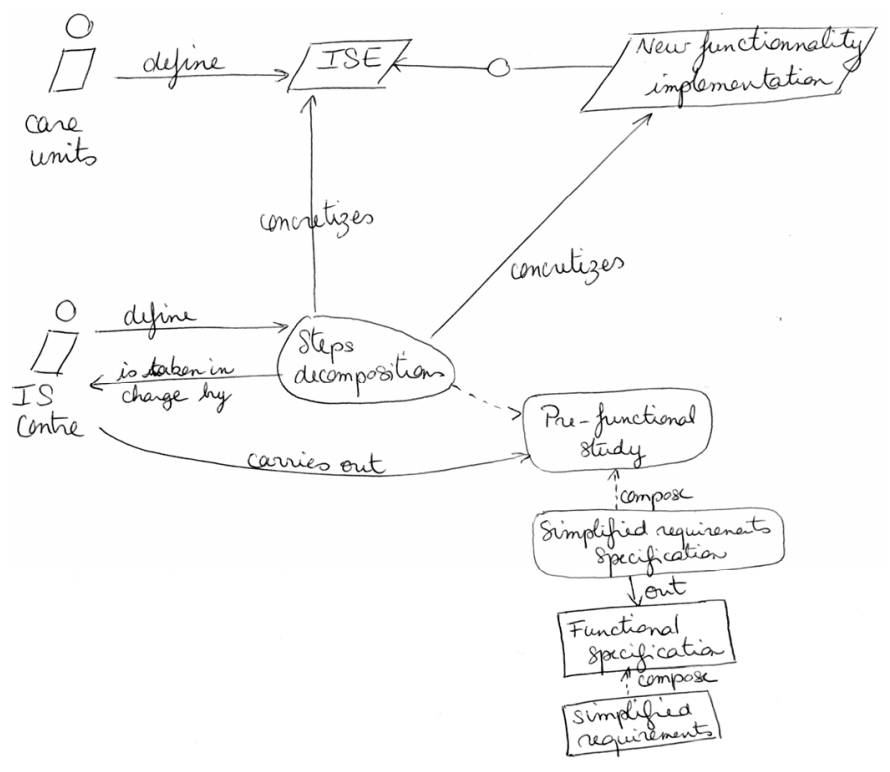

Figure 11. Process model instantiated by a pair of subjects.

As shown in Figure 10, the subjects had carried out 3 iterations between the conceptual graph/process metamodel under construction and the process model. During the first iteration, this pair of subjects selected 8 concepts, during the second iteration, they selected 1 concept and added 2 relations and in the last iteration, they selected 1 concept. Each pair of subjects performed about 3 iterations; hypothesis (6) is validated.

The process metamodel/process model virtuous circle allowed the subjects to "add other concepts" (s.1) or "erase some useless concepts" (s.4): 6 subjects out of 7 (3 subjects did not answer) agreed on the usefulness of the innovation capacity thanks to the iterations. Hypothesis (7) is partly validated.

\section{3) Usability of the method}

The items in Figure 12 allowed us to evaluate the usability of the method.

What did you think of this exercise?

What was the easiest to do?

What was the hardest to do?

Concerning the exercise you did, you are: very satisfied, satisfied, rather not satisfied, no satisfied at all?

Was the method satisfying in relation to the obtained results: yes indeed, yes, not really, not at all?

Can the method avoid you doing mistakes?

Do you think the method can help you to save time? Why?

Do you think the use of the method needs a learning phase?

Do you think this learning phase requires: a few minutes, a few hours, a few days, a few weeks, longer?

Figure 12. Items about the usability of the method.

The method enables to save time ( 9 subjects out of 10,1 without opinion), in particular for a framing of the problem and to avoid doing mistakes during the process model design.

9 subjects out of 10 thought that training on the conceptual graph and the method is necessary, although, a few hours 
would be enough (8 subjects): "This method can be learnt quickly, we can rapidly be operational. I will not spend 15 days to produce a model" (s.2). Hypothesis (4) is then validated.

\section{4) Summarization}

Table II presents the balance of the objectives we wanted to measure, the corresponding hypotheses and the strong and weak points observed during the experiment.

TABLE II. SUMMARIZATION

\begin{tabular}{|c|c|c|c|}
\hline $\begin{array}{l}\text { Objectives to } \\
\text { measure }\end{array}$ & Hypotheses & Strong points & Weak points \\
\hline $\begin{array}{l}\text { Understanding } \\
\text { of the } \\
\text { conceptual } \\
\text { graph }\end{array}$ & $\begin{array}{l}\text { The subjects } \\
\text { will build the } \\
\text { same } \\
\text { conceptual } \\
\text { graph as our. }\end{array}$ & $\begin{array}{l}\text { Concepts have } \\
\text { been correctly } \\
\text { grouped }\end{array}$ & $\begin{array}{l}\text { Relations have } \\
\text { not been } \\
\text { correctly } \\
\text { understood. }\end{array}$ \\
\hline \multirow{4}{*}{$\begin{array}{l}\text { Usage of the } \\
\text { method }\end{array}$} & $\begin{array}{l}\text { The conceptual } \\
\text { graph is useful } \\
\text { to build } \\
\text { information } \\
\text { systems } \\
\text { engineering } \\
\text { process } \\
\text { metamodels. }\end{array}$ & $\begin{array}{l}\text { The process } \\
\text { metamodel is } \\
\text { essential to get } \\
\text { quality process } \\
\text { models. }\end{array}$ & $\begin{array}{l}\text { All the concepts } \\
\text { were not easy to } \\
\text { select. }\end{array}$ \\
\hline & $\begin{array}{l}\text { The conceptual } \\
\text { graph covers } \\
\text { the main } \\
\text { concepts of } \\
\text { information } \\
\text { systems } \\
\text { engineering } \\
\text { process. }\end{array}$ & $\begin{array}{l}\text { The cover of } \\
\text { the concepts is } \\
\text { complete. }\end{array}$ & $\begin{array}{l}\text { Some relations } \\
\text { are missing. }\end{array}$ \\
\hline & $\begin{array}{l}\text { Many iterations } \\
\text { are needed to } \\
\text { get a complete } \\
\text { process } \\
\text { metamodel }\end{array}$ & $\begin{array}{l}2 \text { or } 3 \text { iterations } \\
\text { are needed. }\end{array}$ & \\
\hline & $\begin{array}{l}\text { The virtuous } \\
\text { circle process } \\
\text { metamodel/proc } \\
\text { ess model } \\
\text { allows } \\
\text { innovation }\end{array}$ & $\begin{array}{l}6 \text { subjects think } \\
\text { the iterations } \\
\text { allow } \\
\text { innovation }\end{array}$ & $\begin{array}{l}1 \text { subject thinks } \\
\text { it does not. }\end{array}$ \\
\hline $\begin{array}{l}\text { Usability of the } \\
\text { method }\end{array}$ & $\begin{array}{l}\text { The conceptual } \\
\text { graph is easy to } \\
\text { use and is } \\
\text { efficient }\end{array}$ & $\begin{array}{l}\text { All the subjects } \\
\text { are satisfied of } \\
\text { the method and } \\
\text { the models } \\
\text { designed. }\end{array}$ & \\
\hline
\end{tabular}

The conducted experiment and the results are positive as every hypothesis has been partially or completely validated and no important drawbacks have been detected. It was interesting to divide the experiment in two parts at one week interval. The re-validation of the definitions after the experiment allowed us to propose a better support for the concepts selection.

Although these results are positive, there are still some items to improve. First, the number of subjects did not allow us to carry out a quantitative evaluation of these experiments. We also have to conduct the equivalent experiment with a new focus group that will have the same objective without having the method to help them. This evaluation will really help us to measure the actual effectiveness and efficiency of our method. The prototype was not ready to be used when we conducted the experiment. A next experiment could be conducted in order to measure the perceived ease of use and perceived efficiency of the method via the use of the ProMISE prototype. Finally, the subjects had mostly an academic or research background; as our method is aimed at method engineers, next experiment should include non-academic subjects in order to get more opinions anchored in the field work.

\section{INDUSTRIALISTS EXPERIMENT}

The second experiment has been conducted with nine "industrialists" working in information systems engineering, software engineering and quality. Here are the industrialist's characteristics:

- $\quad$ Average age: 39 years old $(\min 28, \max 49)$,

- Sex: 2 women, 7 men,

- Post: 2 executives, 2 project managers, 1 quality engineer, 1 software engineer, 1 IT manager, 2 programmer-analysts,

- Company size average: 1200 employees (min 1, max 7300).

It is important to underline the fact that industrialist's experiments are costly in terms of information collection and analysis; however it is the richest method to get the largest range of opinions and perceptions.

\section{A. Objectives}

The objectives of this experiment were to know:

- The industrialists' habits in terms of information systems or software methods and their setting-up in their organization,

- The industrialists' opinion about our method, in terms of usage and usability of the method and usability of the prototype.

Due to the lack of free time of the industrialists, it was hard to organize long and complex experiments. So, these experiments were conducted as demonstrations and interviews that allow efficiently and quickly collecting opinions. It was not about quantifying the answers but collecting the more different opinions as possible.

\section{B. Hypothesis}

Table III details the different objectives and the corresponding hypotheses of this experiment.

\section{TABLE III. OBJECTIVES AND HYPOTHESIS}

\begin{tabular}{|c|c|}
\hline $\begin{array}{l}\text { Objectives to } \\
\text { measure }\end{array}$ & Hypotheses \\
\hline \multirow[b]{2}{*}{ Usage of the method } & The method is useful to build process models (1) \\
\hline & $\begin{array}{l}\text { Creating the process metamodel is useful to get } \\
\text { the process models ( } 2 \text { ) }\end{array}$ \\
\hline $\begin{array}{l}\text { Usability of the } \\
\text { method }\end{array}$ & The method is easy to use and is efficient (3) \\
\hline $\begin{array}{l}\text { Usability of the } \\
\text { prototype }\end{array}$ & The prototype is easy to use and is efficient (4) \\
\hline
\end{tabular}


The focus group experiment and the industrialists experiment were conducted in a short time interval, the industrialists experiment was then conducted disregarding the feedback from the focus group experiment.

\section{Protocol}

Each subject was met individually, as a majority on their workplaces. The experiment was divided in six small parts where we alternatively orally asked questions and presented slides. In the first part, we asked them about their work habits concerning the use of methods for information systems/software engineering in their organization. The answers were very instructive and sometimes astounding. The answers are presented in detail in [3].

In the second part of the experiment, we presented an example of metamodelling and interrogated the subjects about it. In the third part, we presented them the method and the conceptual graph and asked their opinion about them. In the fourth part of the experiment, we presented a demonstration of the prototype with the selection of the concepts and the visualization of the conceptual graph. In the fifth part, we presented the process metamodel under construction corresponding to the selected concepts within the prototype. The last part consisted of presenting the selection of a formalism and the instantiation of the process metamodel using the defined formalism.

The experiment lasted between one hour and a half and two hours, according to the available time and the loquacity of the subjects.

\section{Results}

We describe here the feedback received from the industrialists.

\section{1) Method}

Figure 13 presents the items about the method and the conceptual graph.

What do you think about the approach and the different points of view?

What do you think of the intentional level?

What do you think of the operational level?

What do you think of the concepts?

What do you think of the relations?

Figure 13. Items about the method and the conceptual graph.

All the subjects considered the points of view interesting: "they are very adequate" (s.3), "all these points of view are principles we can take advantage of. They cover and stick well with the principle we want to apply" (s.6), "It allows to learn rapidly with a referential" (s.2), "What is good with these points of view is to see how they imbricate with each others" (s.9).

The subjects recognized their work in the activity oriented point of view: "We see that on the schedules, it is the kind of visual notation we are used to see" (s.4).
The product oriented point of view was also well known: "We exchange documents that we version. The internal or customer validation changes the state of the document" (s.4).

The decision oriented point of view was familiar: "It's the methodology problem-solution used in my company" (s.1), "Decision oriented modelling is rather classic" (s.2), "The decision aspect can be used to control the drifts of a project" (s.6) but this point of view is not necessarily modelled: "It is never modelled, not even presented on the board when we have problems, we only discuss them" (s.4).

The context oriented point of view is useful: "It's a state of the project of where we are and towards what we want to go" (s.1), 'It's at the throwing meeting: that's where we are, that's what we want to get. At the closure meeting: what have been got or not. It is only verbal" (s.4).

All the subjects agreed that the strategy oriented point of view concerns the decision-makers: "I do not establish [the strategies], I only follow them. The project leaders, the direction team fix them" (s.4), "The strategy is a project execution plan, objectives not very detailed, defined by the project or program managers" (s.6), "Strategy is for the buyer" (s.1), "Strategy is political: it will orient what we want to do, it is given by the information systems direction" (s.8).

The abstraction levels, intentional and operational, are recognized by the subjects. The intentional level is aimed to the leaders: "It is the commercial level" (s.1), it represents "what we would like to do. It can be influenced by the company's strategy. It is not entirely fixed by the developer or project manager" (s.2), "The project manager has integrated the customer request, has proposed the adapted solution, he/she knows the intentions and coordinates the development tasks. He/she gives the actions to set up. It's his/her responsibility" (s.4).

The operational level corresponds to "what we really do" (s.3), "It is our responsibility (programmer-analysts): the actions to carry out within time and the needed deliverable" (s.4).

Both levels are "useful, I see both levels in project management. That's why we have different levels of responsibilities: those who carry out the actions, those who are responsible and define the objectives" (s.6).

The results obtained in this part of the interview supports the theoretical proposition of the method in terms of points of view and abstraction levels.

\section{2) Construction of the process metamodel}

We first presented the part of the conceptual graph representing the path of the selected concepts. Figure 14 presents the items about the conceptual graph.

What do you think of the conceptual graph?

Could it help you to understand metamodelling? Why?

Would you use it? What would you use it for?

Figure 14. Items about the conceptual graph.

The subjects had different opinions about the conceptual graph presented in the prototype. Some of them found an 
interest in the conceptual graph it-self, independently from the method: "the user has a referential" (s.2), "it forces us to ask ourselves questions" (s.7), "I think it could help, it's ready to use, interactive and it allows browsing in the metamodelling concepts" (s.6).

The visual aspect of the conceptual graph is important: "If the customer needs a formalization of his/her habits, the tool is structuring enough. It allows having a comprehensible result, graphical, even if it could be hard to get for a non-computer specialist" (s.2), "It's a visual support, it's easily accessible" (s.3).

For others, it was hardly understandable because it is too abstract: "We need the model to better understand the metamodel" (s.4), "It could be interesting to apply it to something more concrete, that is to say at a lower modelling level" (s.7), "It stays in the abstraction" (s.1), "For me, it's too abstract, now, we do not ever manage to model at a lower level" (s.8).

The subjects underlined the fact that the conceptual graph is a first step to go further in process modelling: "We cannot give that to the users. Once we understand this domain, we have to produce something that can be useful for the users" (s.6), "I need an instance of this graph" (s.3), "The model level is missing" (s.4), "It must be a step that leads us to propose a more concrete level to propose to the users" (s.6), "If I can go one level lower, I'll use the tool" (s.7), "It the expectations are more practical, a link is missing with existing process modelling tools in order to share the same concepts, to set up the following of the process" (s.2), "I would be interested if I could instantiate the lower levels. If it could give me a file in a usable format by tools able of instantiating processes. On the fieldwork, I would like something concrete, where we can generate a kind of website where people collaborate on the same process. I can do the abstraction effort but it needs to be concretized. Metamodelling is ok but we absolutely need to instantiate it and put it in the situation" (s.9).

At last, some subjects gave suggestions to improve the prototype: "Method engineers could annotate the concepts they selected to justify, to cross the information" (s.2), "I would work by deleting the concepts in the conceptual graph or using a check-list or priorities on the concepts" (s.1).

The conceptual graph is then useful but only if it can lead to design process models, which is the purpose of our method.

The metamodel was then presented on the form of a class diagram. Items about it are presented in figure 15.

What do you think of the process metamodel?

In comparison to the conceptual graph, what do you think?

Which diagram do you prefer? Why?

Figure 15. Items about the process metamodel.

Some subjects pointed that UML is hard to understand: " $U M L$ is inappropriate as dialog language, it's too complicated; we need something easier" (s.1), "The metamodel will scare off non-computer specialists. The conceptual graph is more reassuring" (s.2), "Everybody does not have the same comprehension level of the metamodel, if there is no example, its difficult" (s.4).
Some subjects underlined the interest of the conceptual graph in comparison to the class diagram: "the functional knowledge is not always exploited by the computer specialists; there are always people who have specific knowledge and could understand the conceptual graph but could not understand the metamodel as a class diagram" (s.2).

Nevertheless, the class diagram is more accurate than the conceptual graph: "There is some interesting information; the class diagram is richer, more detailed than the conceptual graph" (s.2), "In UML, there is formalism for the input and output of the activities" (s.6).

The complementarity between the conceptual graph and the class diagram was pointed out: "It's good to reach one from another" (s.1), "It's fine to have both because we don't understand everything at first sight. They are very complementary" (s.3), "Both give a different point of view: in the conceptual graph, there are rather principles. UML has a more detailed formalism" (s.6).

The majority of the subjects prefer nevertheless the class diagram than the conceptual graph ( 7 subjects out of 9 ).

3) The method in its entirety and the prototype

The last part of the experiment was the presentation of the instantiation and the prototype. The subjects could then evaluate the method in its global nature and the prototype (items presented in figure 16).

Do you think this method can help you in the definition of your process models? Why?

Do you think the prototype can avoid you doing mistakes? Why?

Does the proposed formalism seem useful to you? Interesting?

In the proposed method, the metamodel is built before the model, what do you think about that?

Is it a usual way of building models for you?

Figure 16. Items about the method within the prototype.

Subjects thought that the method and the prototype could help them in the definition of their process models: "The method will allow me to select the vocabulary with which I will express the vision of the processes" (s.4), "There's an interesting visual support. The fact that it is computerized allows not scribbling and loosing sheets" (s.3), "The tool can help me to have more flexibility, to have more models at my disposal to better implement the flows" (s.6). The method can be used to "Validate the whole processes that are conform to the metamodel and those which are not. It's useless to do a metamodel if only 50\% of the processes are conformed. The metamodel has to be attached to all the already modelled processes. Companies are growing, they need more formal methods, they do the effort to model, or for the setting up of the ISO, to model their activities to estimate the quality of this modelling" (s.2). "The tool is practical and close to the field" (s.1), "The advantage of the metamodel as it is proposed is the fact that we can use more organizational and strategic aspects. We can have different facets of a process to have something very consistent" (s.9). Hypothesis (1) is then validated.

The prototype also allows defining useful models to communicate: "The work documents to exchange are nice" (s.1), "It can be a communication support, including the 
direction that does not have the precise knowledge of what people do, but gives a global vision of the information systems part. It's something quite visual and easy to understand" (s.7).

The prototype can also help method engineers to avoid doing mistakes: "It helps us to efficiently model, it gives us enough categories to reuse in the processes. It is rich enough" (s.6), "It avoids introducing concepts that are not pertinent, it allows to structure" (s.2). A part of the mistakes can be avoided in the process models thanks to the metamodel defined higher up the line: "since I have well defined my metamodel" (s.3), "if there is a metamodel behind" (s.4), "the instantiation of the metamodel will allow generating something consistent. Conversely, the metamodel instantiation allows validating it" (s.9). Hypothesis (2) is then validated.

Nevertheless, a subject insisted on the notion of check-list: "if there is a check list, it avoids forgetting concepts" (s.1) and another subject brought back the problem of "gap between the modelled activity and the real activity" (s.5).

Being able to select his own formalism is interesting; "it is part of the tool flexibility" (s.6), and can be useful in "big companies, they must have homogenization of their models" (s.7).

The approach we propose goes from the process metamodels to the models. Some subjects would prefer the opposite approach, from the models to the metamodel: " $A$ lot of companies already have defined process models. It is easier for a method engineer to select concepts in relation to already defined models. It's cyclic: construction of the M1 then M2" (s.2), "from one or more models at M1 to do the M2" (s.5). The metamodelling or even the modelling is however not a habit for all the subjects, which could explain why the approach is not natural: "I rarely model" (s.4), "It's the first time, I will experiment level M2, I have always worked at MO and MI" (s.6), "I directly work at M1." (s.7)

The method and the prototype would help the subjects to "structure the communication with the customer, explain where his role is in the process and to transmit knowledge" (s.1), to "define an ideal process metamodel of a company; it is an interesting element for optimization. If we have difficulties to model the processes with the tool, it could mean that there are dysfunctions in the processes, incoherence or deadlocks. The method will allow identifying, thinking, underlining incoherent processes" (s.2) or to "define processes at a very high level to propose them to the customer, integrate customer's process in a repository, to help them improve their work methods. As a consultant, it is also my role: in intern to define our process and compare them to the customer's and for the customers, implement and follow them" (s.3).

The need of adaptation to the domain is often cited: " $A$ model like that has to be the more generic as possible, but there are specificities linked to the companies business. It is necessary to do tailorisation in relation to the business domain" (s.6), "I don't know if we would obtain a sufficiently generic result, but not too generic" (s.2).

Figure 17 presents some items about using the method.

Would you be ready to use this method in your work environment?
Would you advise the use of the method?

Would you be ready to describe the method to another person?

Figure 17. Items about using the method.

7 subjects out of 9 would be ready to use the method in their work environment. 2 out of 9 would not use it because they do not use method or because the method is too abstract: "We tried Enterprise Architect, it worked with people who knew Object Oriented Design, with young people but not with the others. Even with those whom it worked, it did not last. It does no fit with our context" (s. 8).

6 subjects out of 9 would advise the use of the method because "it is structuring" (s. 2), and "If you want your process models to obey rules, try to do it using these concepts and these relations, it will avoid you doing mistakes or creating incoherent or unenforceable models" (s. 4).

Then, we asked questions about the method's learning (see figure 18).

Do you think the use of the method needs a learning phase?

Do you think this learning phase requires: a few minutes, a few

hours, a few days, a few weeks, longer?

Figure 18. Items about the learning of the method.

All the subjects (9 out of 9) thought that the use of the method and the prototype needs a learning phase. The appropriation phase could last a few hours for people who know metamodelling to one week for the others. Two subjects underlined the fact that class's diagrams will be understood with difficulty by non-computer specialists: "For the decisionmakers in companies who are not familiar with UML, it will be hard" (s.3), "A decision-maker will model quite quickly because it is intuitive but metamodelling, never!" (s.7)

Finally, we asked some questions about the prototype ease of use.

Is this prototype pleasant to use?

Would you need help during the use of the prototype?

Is the interface of the prototype clear and easy to understand?

Figure 19. Items about the prototype.

Concerning the interface of the prototype, some subjects noticed its lack of ergonomics. Furthermore, it is important for the prototype to allow the execution and the following of the process: "All we do must be useful for someone and for something. The goal is to give the users a tool that is efficient to describe their project, to use it and execute it" (s.6).

To conclude, we can say that hypothesis (3) and (4) are partly validated as the method is not natural for every body and the prototype can be improved.

\section{4) Summarization}

Table IV presents the synthesis of the objectives we wanted to measure, the subjacent hypotheses and the strong and weak points recorded during the industrialist's interviews.

TABLE IV. SUMMARIZATION

\begin{tabular}{|c|c|c|c|}
\hline $\begin{array}{c}\text { Objectives } \\
\text { to measure }\end{array}$ & Hypotheses & Strong points & Weak points \\
\hline
\end{tabular}




\begin{tabular}{|l|l|l|l|}
\hline $\begin{array}{l}\text { Objectives } \\
\text { to measure }\end{array}$ & \multicolumn{1}{|c|}{ Hypotheses } & \multicolumn{1}{|c|}{ Strong points } & \multicolumn{1}{|c|}{ Weak points } \\
\hline & $\begin{array}{l}\text { The method is } \\
\text { useful to build } \\
\text { process models } \\
\text { concepts is } \\
\text { complete at first } \\
\text { sight. }\end{array}$ & $\begin{array}{l}\text { - The method } \\
\text { avoids doing } \\
\text { mistakes. } \\
\text { - The formalism } \\
\text { is useful. }\end{array}$ & $\begin{array}{l}\text { - Metamodelling } \\
\text { is too abstract for } \\
\text { some subjects. }\end{array}$ \\
$\begin{array}{l}\text { Usage of the } \\
\text { method }\end{array}$ & $\begin{array}{l}\text { Creating the } \\
\text { process } \\
\text { metamodel is } \\
\text { useful to get } \\
\text { the process } \\
\text { models (2) }\end{array}$ & $\begin{array}{l}\text { - The metamodel } \\
\text { avoids doing } \\
\text { mistakes in the } \\
\text { process models. }\end{array}$ & $\begin{array}{l}\text { - It's more natural } \\
\text { to first create the } \\
\text { process models. }\end{array}$ \\
\cline { 2 - 5 } $\begin{array}{l}\text { Usability of } \\
\text { the method }\end{array}$ & $\begin{array}{l}\text { The method is } \\
\text { easy to use and } \\
\text { is efficient (3) }\end{array}$ & $\begin{array}{l}\text { - Learning phase } \\
\text { would be short } \\
\text { for people who } \\
\text { know about } \\
\text { metamodelling. }\end{array}$ & $\begin{array}{l}\text { - Metamodelling } \\
\text { is hard for non } \\
\text { computer } \\
\text { specialists. }\end{array}$ \\
\hline $\begin{array}{l}\text { Usability of } \\
\text { the prototype }\end{array}$ & $\begin{array}{l}\text { The prototype } \\
\text { is easy to use } \\
\text { and is efficient } \\
\text { (4) }\end{array}$ & $\begin{array}{l}\text { - The prototype is } \\
\text { inline with the } \\
\text { method. }\end{array}$ & $\begin{array}{l}\text { - The interface } \\
\text { could be more } \\
\text { user-friendly. }\end{array}$ \\
\hline
\end{tabular}

The industrialist's interviews allowed pointing up, on the one hand, the need of efficient process modelling tools, and on the other hand, the necessity to execute and follow these processes. Our method and the subjacent prototype partly fulfil the expressed difficulties and needs. The concepts we propose and their assembly allows obtaining a structured and structuring result. Many perspectives have been addressed to improve the prototype.

\section{5) Critics}

The industrialist's interviews were conducted when the method and the prototype were in their nearly final version. We were not able to carry out these experiments before as we did not have the required means and resources to properly set up a protocol four years ago.

Ideally, general interviews should have been done at the beginning of the project, in order to evaluate the interest of metamodelling and points of view for industrialists. According to the result, the method would have been developed and presented to the focus group. Once its validation, we could have presented it to the industrialists for a mid-term evaluation. A final version of the method would have then been carried out and evaluated by the industrialists.

Generally speaking, a method can not be completely evaluated in a few hours. The users need to assimilate it and regularly use it in order to get deeper and more tangible results.

\section{CONCLUSION}

In this paper, we described a qualitative evaluation of a method for information systems engineering processes. We exposed the different objectives according to the subject's profiles (focus group and industrialists) and the hypotheses we had to verify. According to the hypotheses, different questions were asked to the subjects. We also presented the results of this evaluation that allowed us to improve the method.

The strong points of this evaluation are:

- A two phases experiment for the focus group: this allowed a better assimilation of the concepts and therefore demonstrated that the learning phase of the method is short.

- The feed-back we proposed to the focus group after the experiment in order to validate the new version of the concepts and relations definitions: this double evaluation ensures that the definitions are better understandable by method engineers.

- The industrialist's qualitative interviews: expensive in terms of opinions collection and analysis, it permits us to get a rich range of opinions and perceptions of the method and the prototype.

We focused on the qualitative aspect of evaluation; we now have to build experiments in order to measure the quantitative aspects of the method. These experiments will concern the measures of inputs and outputs, as specified in the Methodological pragmatism [7], that is to say on the duration needed to carry out a process model according to different configurations (with or without the method, with or without the prototype), and on the quality of the process models obtained according to the configurations.

\section{ACKNOWLEDGMENT}

We would like to thank all the subjects that participated to the experiments.

\section{REFERENCES}

[1] Collins, English Dictionary 5th Edition, 2000.

[2] ISO, ISO 9241-11 Ergonomic requirements for office work with visual display terminals (VDTs) -- Part 11: Guidance on usability, 1998.

[3] Charlotte Hug, Méthode, modèles et outils pour la méta-modélisation des processus d'ingénierie de systèmes d'information, $\mathrm{PhD}$ thesis, Joseph Fourier University, October 2009

[4] Charlotte Hug, Agnès Front, Dominique Rieu, Brian Henderson-Sellers, A method to build information systems engineering process metamodels. J. of Syst. and Soft. 82, 10 (Oct. 2009), 1730-1742. DOI=http://dx.doi.org/10.1016/j.jss.2009.05.020

[5] Fred D. Davis, Perceived usefulness, perceived ease of use, and user acceptance of information technology. MIS Quarterly, 13, 3 (Sept. 1989), 319-340.

[6] Daniel L. Moody, The method evaluation model: a theoretical model for validating information systems design methods. In Proceedings of the 11th European Conference on Information Systems (ECIS 2003), Naples, Italy.

[7] Nicolas Rescher, Methodological Pragmatism: Systems-Theoretic Approach to the Theory of Knowledge, Basil Blackwell, Oxford, 1977

[8] Jakob Nielsen, Usability Engineering, Morgan Kaufmann Publishers, 1997

[9] Philippe Mallein, Yves Toussaint, Technologies de l'information et de la communication: sociologie pour la Conception Assistée par l'Usage, Communications \& stratégies, Cahiers de l'IDATE, 5, 1994 